\title{
Performance Evaluation of Conflict-Free Trajectory Taxiing in Airport Ramp Area Using Fast-Time Simulations
}

Nikolai Okuniek (DLR)

Yoon Jung (NASA)

Ingrid Gerdes (DLR)

Zhifan Zhu (NASA/SGT)

Sergei Gridnev (NASA/SGT)

Hanbong Lee (NASA)

$37^{\text {th }}$ Digital Avionics Systems Conference September 23-27, 2018 London, UK
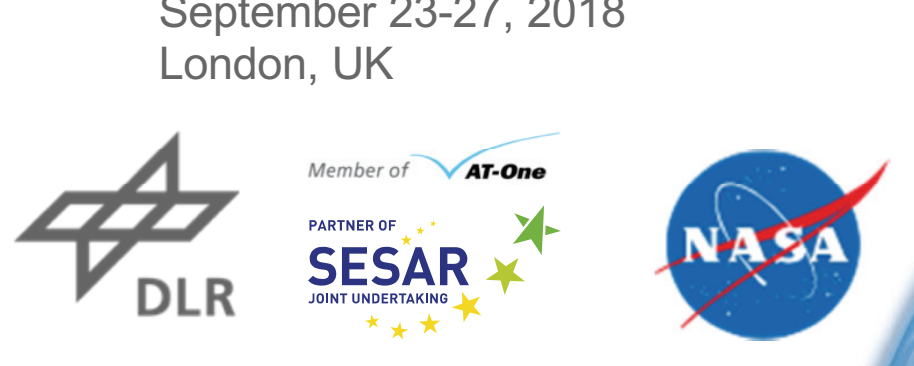


\section{Outline}

- Background

- Research Objective and Technical Approach

- Simulation Setup

- Results and Analysis

- Summary and Future Research

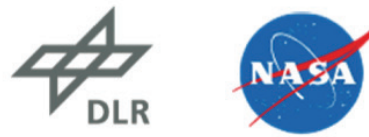




\section{Background - DLR \& NASA Collaboration}

Collaborate in the area of airport surface operations to jointly investigate advanced ATM concepts/tools:

- Started in 2013

- Investigate a harmonized concept of operations for surface operations ${ }^{1}$

- Conduct simulations: independent ${ }^{2}$ and integrated

1. N. Okuniek, et al., "A Concept of Operation for Trajectory-based Taxi Operations," 16th AIAA ATIO Conference, Washington D.C, USA, June 13-17, 2016.

2. Z. Zhu et al., "Performance Evaluation of the Approaches and Algorithms for Hamburg Airport Operations," 35th DASC, Sacramento, CA, USA, September 25-29, 2016. 


\section{Research Objective and Technical Approach}

- Objective: Investigate conflict-free, time-based taxi optimization capability applied to ramp operations using integrated fast-time simulations

- Technical Approach

- Adapt the existing conflict-free surface traffic optimization tool to ramp operations

- Integrate with a fast-time simulation tool

- Develop common performance metrics in efficiency, throughput, predictability, and environmental benefits

- Compare the simulation results with the baseline simulation
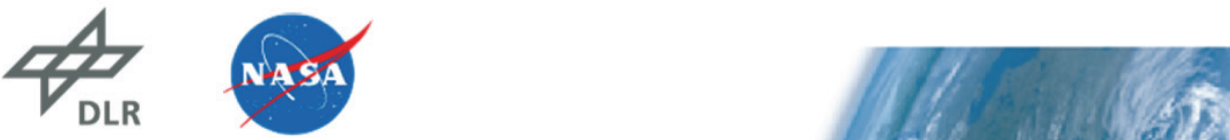


\section{Contributing Technology - Taxi Routing for Aircraft: Creation and Controlling (TRACC)}

- Coupled with a Departure Management System (DMAN) determines the optimal surface movement plans

- Generates conflict-free 4D taxi (4DT) trajectories and calculates Target Start-up Approval Time (TSAT)

- Transfers 4DT trajectories into a speed profile with corresponding advisories for controllers and pilots

- Tested the prototype tool in a human-in-theloop simulation environment

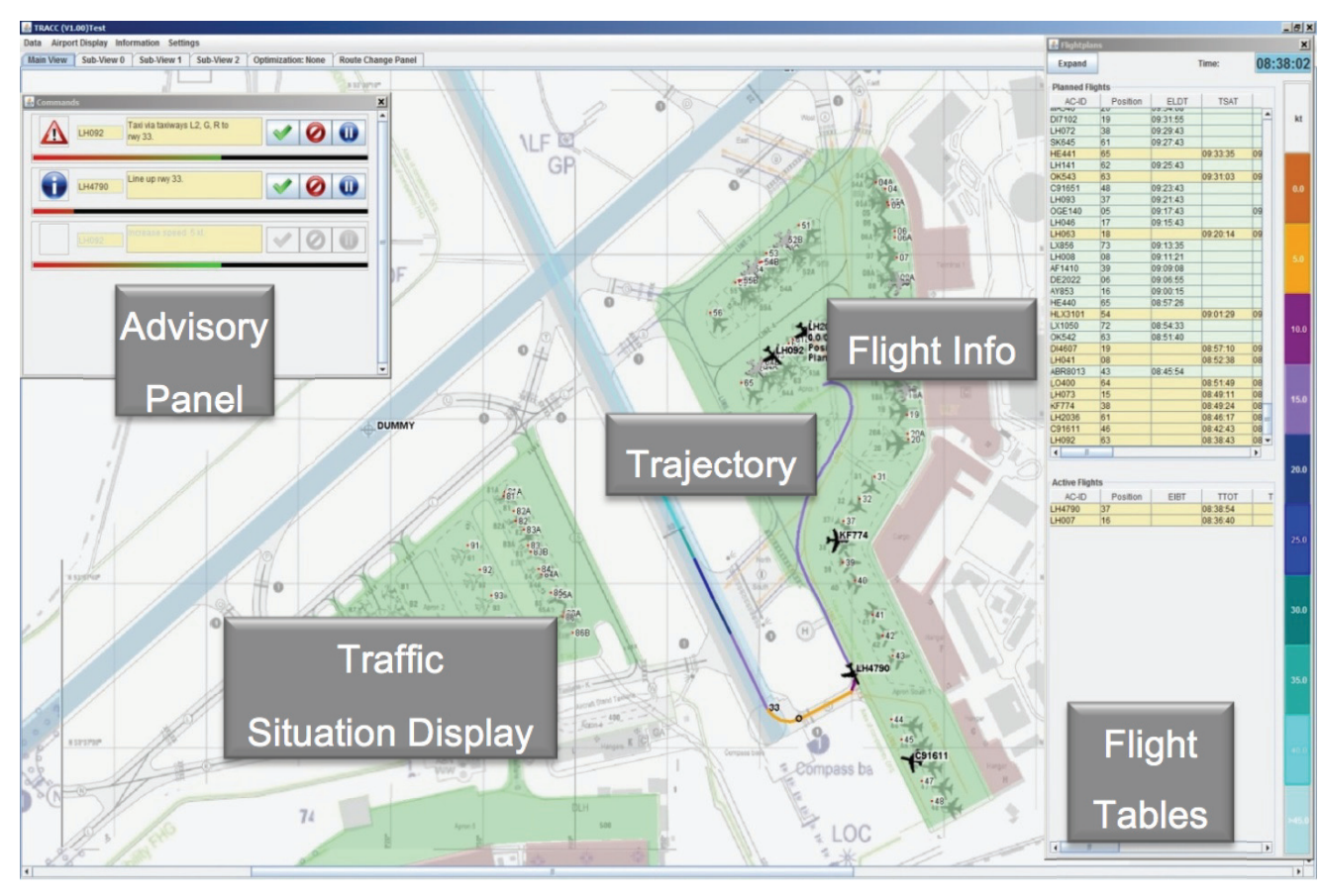

TRACC User Interface for Tower Controller (Hamburg Airport, Germany)
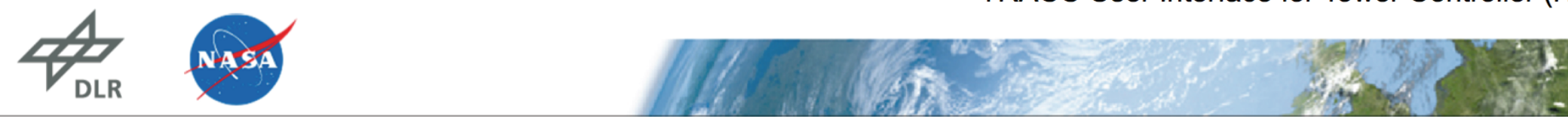


\section{TRACC_PB: TRACC for Pushback Optimization}

- Solve for optimized conflict-free taxi trajectories in the ramp area

- Inputs:

- Prescribed taxi routes

- Scheduled departure times and target movement area entry times for departures

- Target ramp entry times for arrivals

- Outputs:

- Optimized taxi speed profile that satisfies target times for departures and arrivals

- Target pushback times for departures

- Target movement area entry time and target ramp entry time are adjusted if there is no conflict-free trajectory available 


\section{TRACC_PB for Ramp Operations}

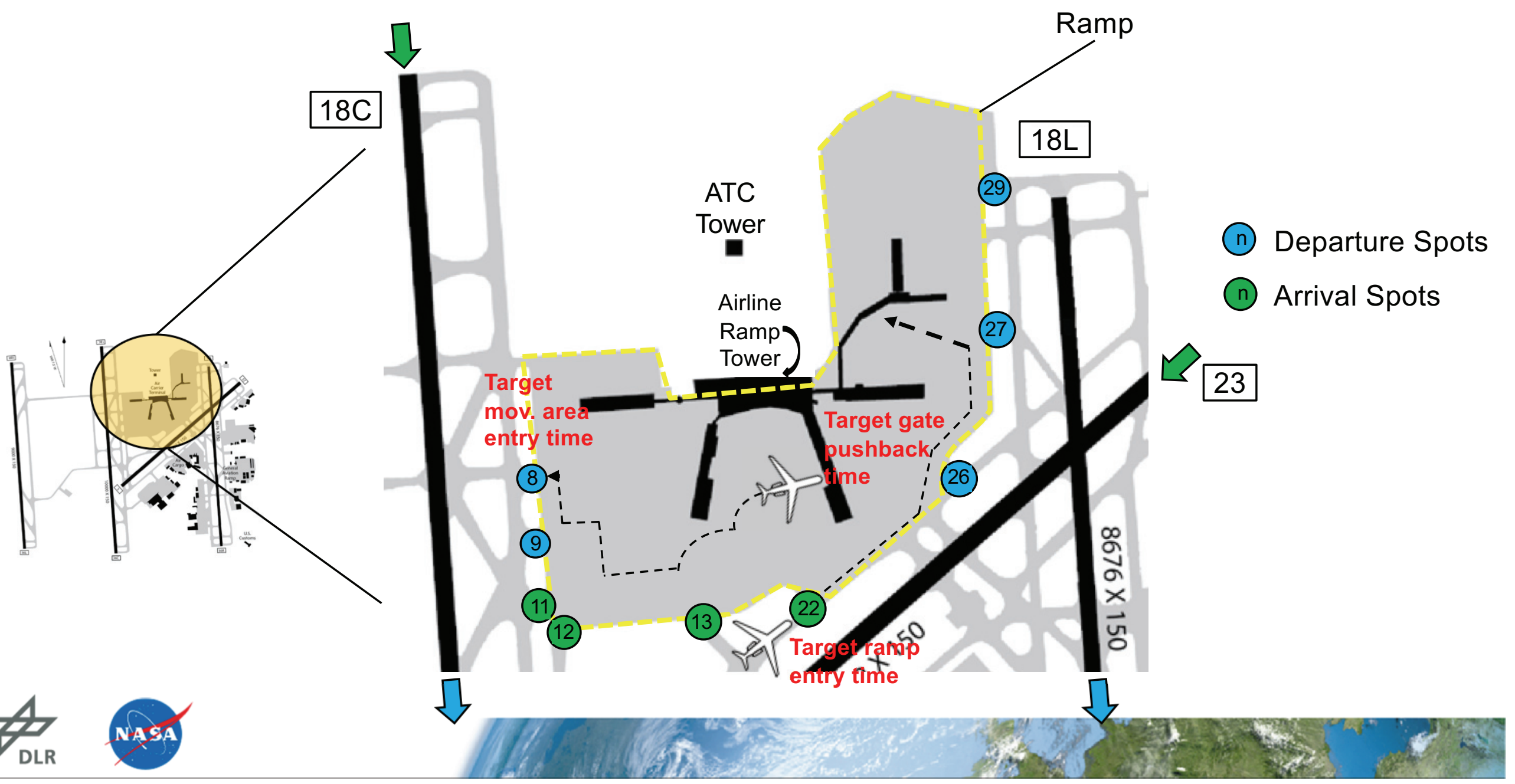




\section{Contributing Technology - Surface Operations Simulator and Scheduler (SOSS)}

- Fast-time airport surface simulation tool based on a node-link model

- Capable of modeling uncertainties, including taxi speeds, pushback process

- Common Algorithmic Interface (CAI) allows for testing schedulers independent of the model

- Has a built-in Conflict Detection and Resolution (CD\&R) function to prevent loss of separation

- Used for development/testing of schedulers that can be plugged in a real-time system
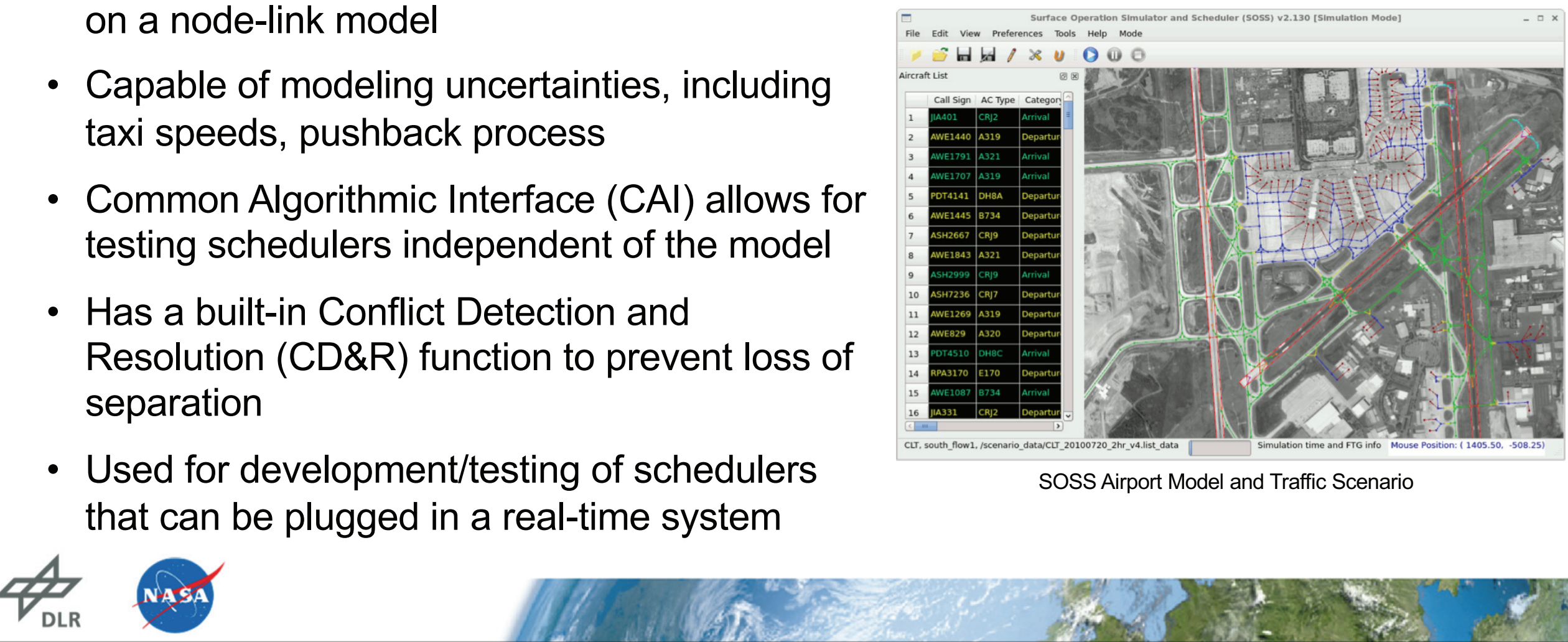


\section{Simulation Setup - Baseline System}

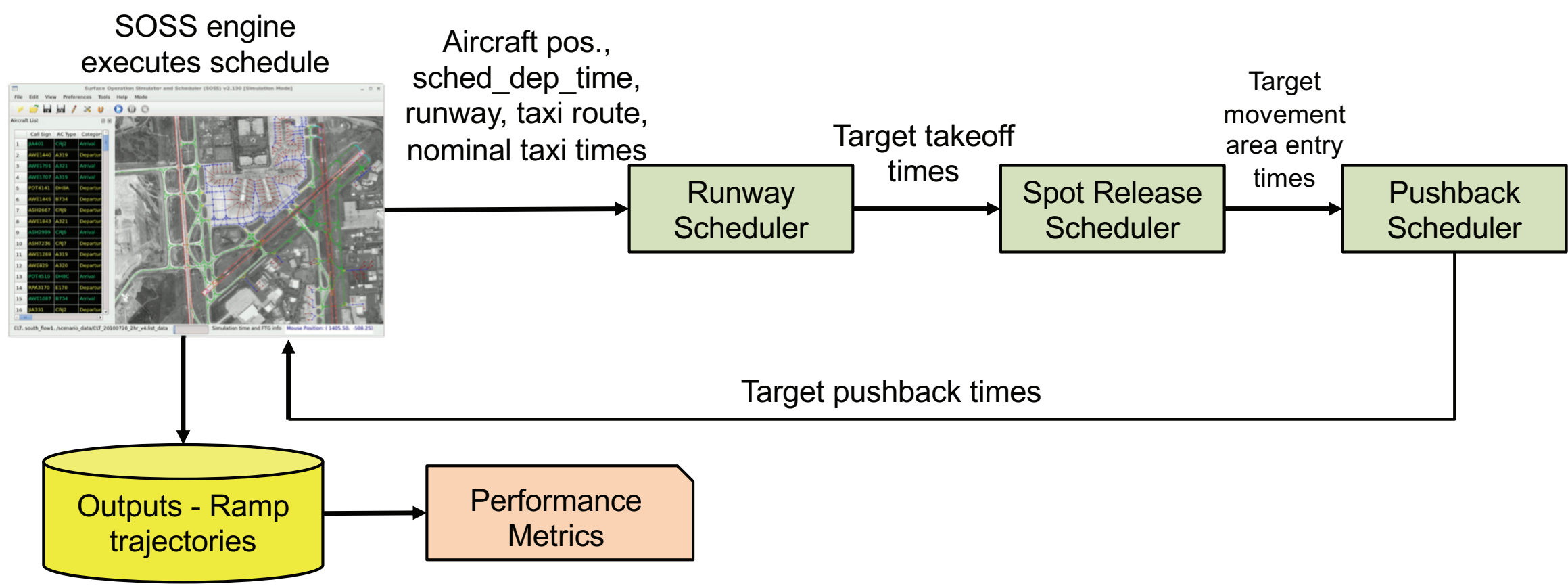

- SOSS CD\&R function is used to maintain separation of traffic on the surface

- Target movement area entry and target ramp entry times are saved for TRACC_PB simulation

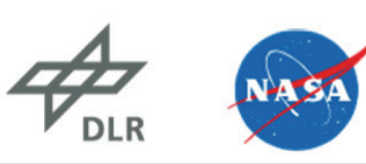




\section{Simulation Setup - TRACC_PB System}

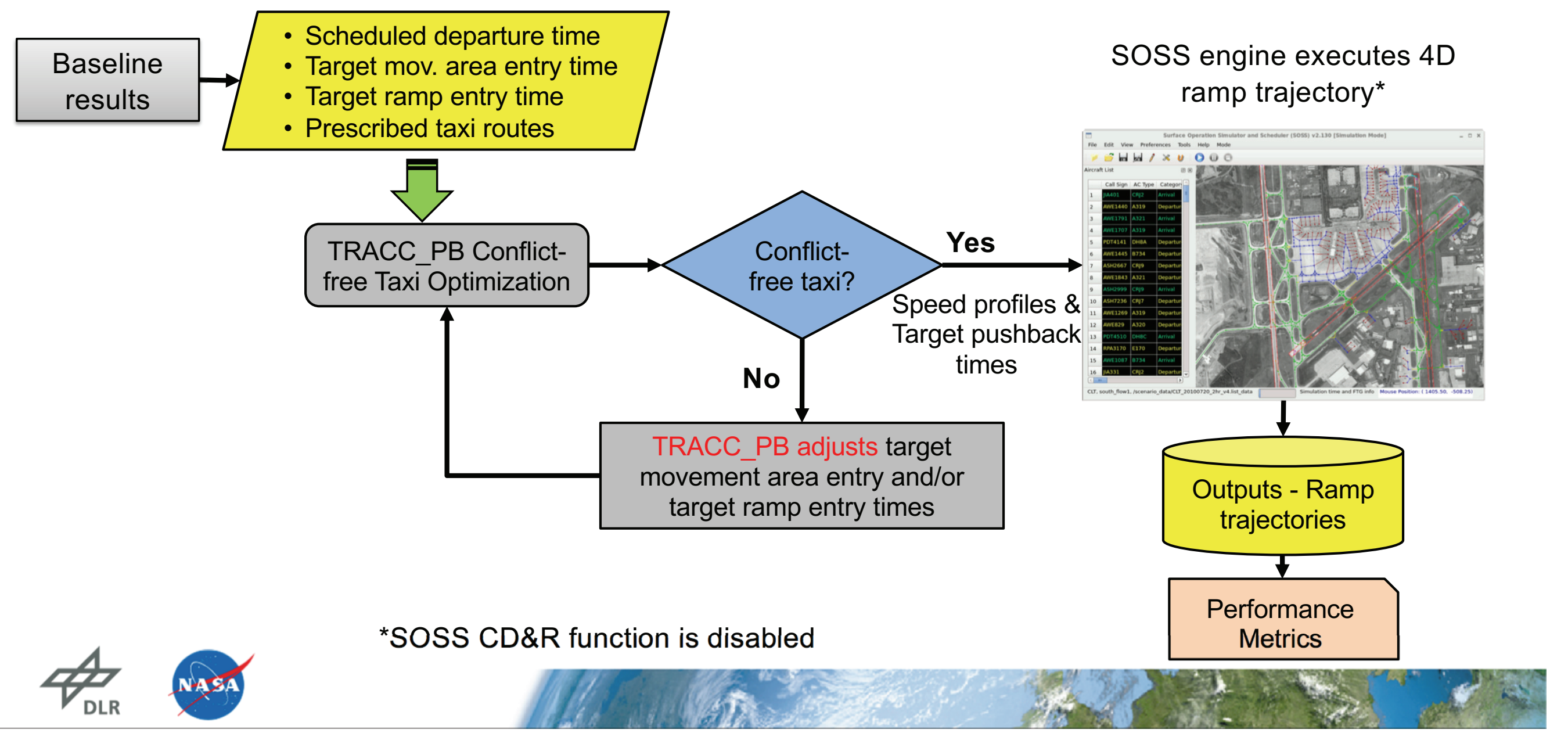




\section{Traffic Scenario}

\section{Airport}

- Charlotte Douglas International Airport (CLT)

- Four runways in a south flow configuration

- RWY 18L (departure only)

- RWY 18R, 23 (arrival only)

- RWY 18C (dual usage)

- Arrival spots: 11, 12, 13, 22

- Departure spots: 8, 9, 26, 27, 29

\section{Traffic Scenario}

- 138 flights (medium traffic density)

- 62 departures, 76 arrivals

- Scenario duration: about 2.5 hours
Departure spots

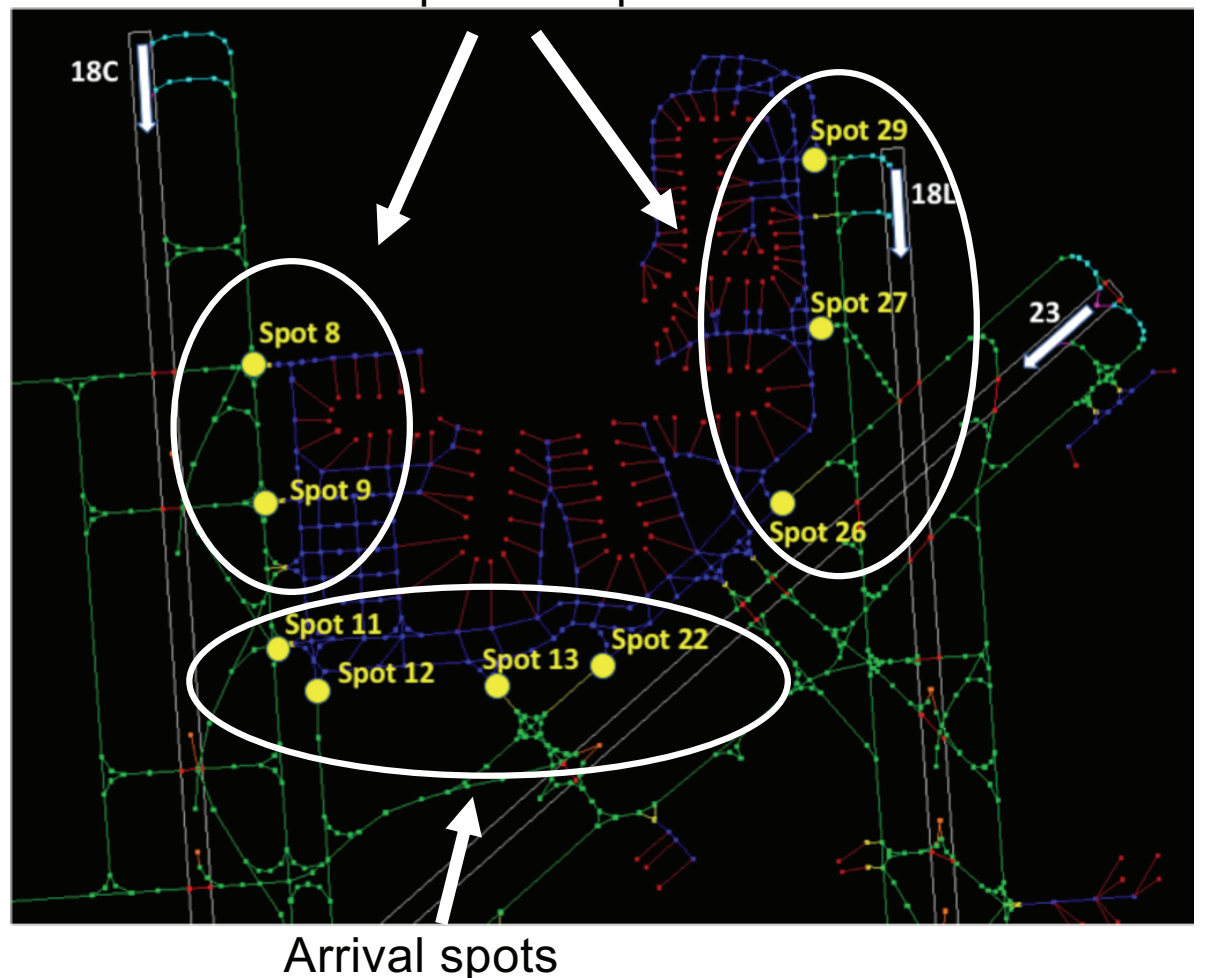

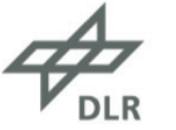




\section{Performance Metrics}

- Ramp throughput

- Ramp Taxi time

- Gate Hold Time

- Ramp Departure Flight Count

- TMAT* Compliance

- Environmental Benefits

"TMAT $=$ Target movement area entry time

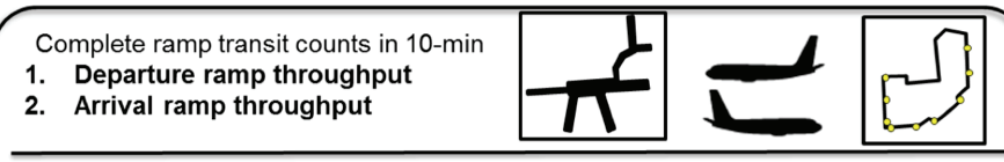

Number of flights in ramp area taxiing to their assigned spots at the same time

6. Ramp Departure Flight Count

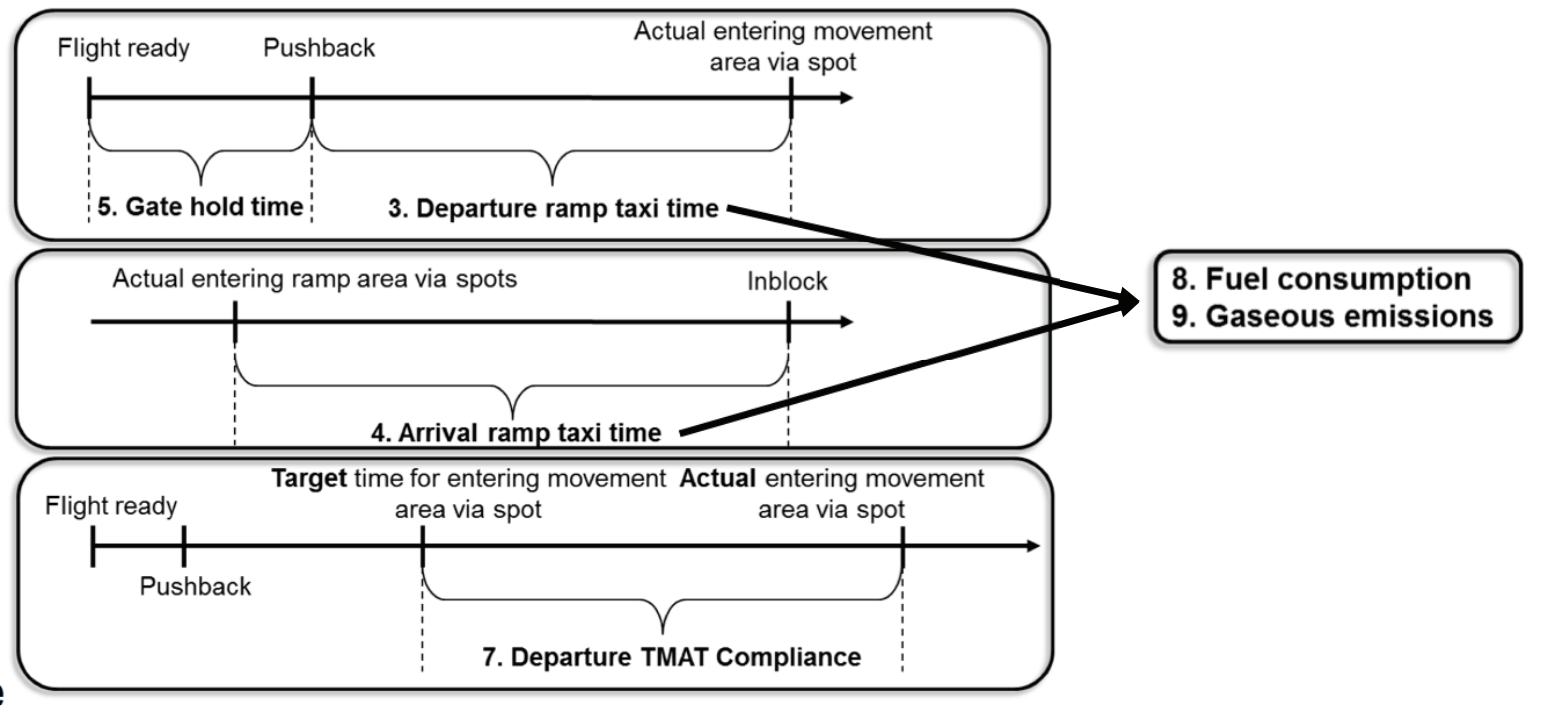

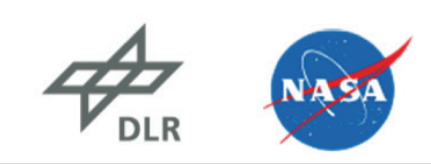




\section{Results and Analysis - Ramp Throughput}

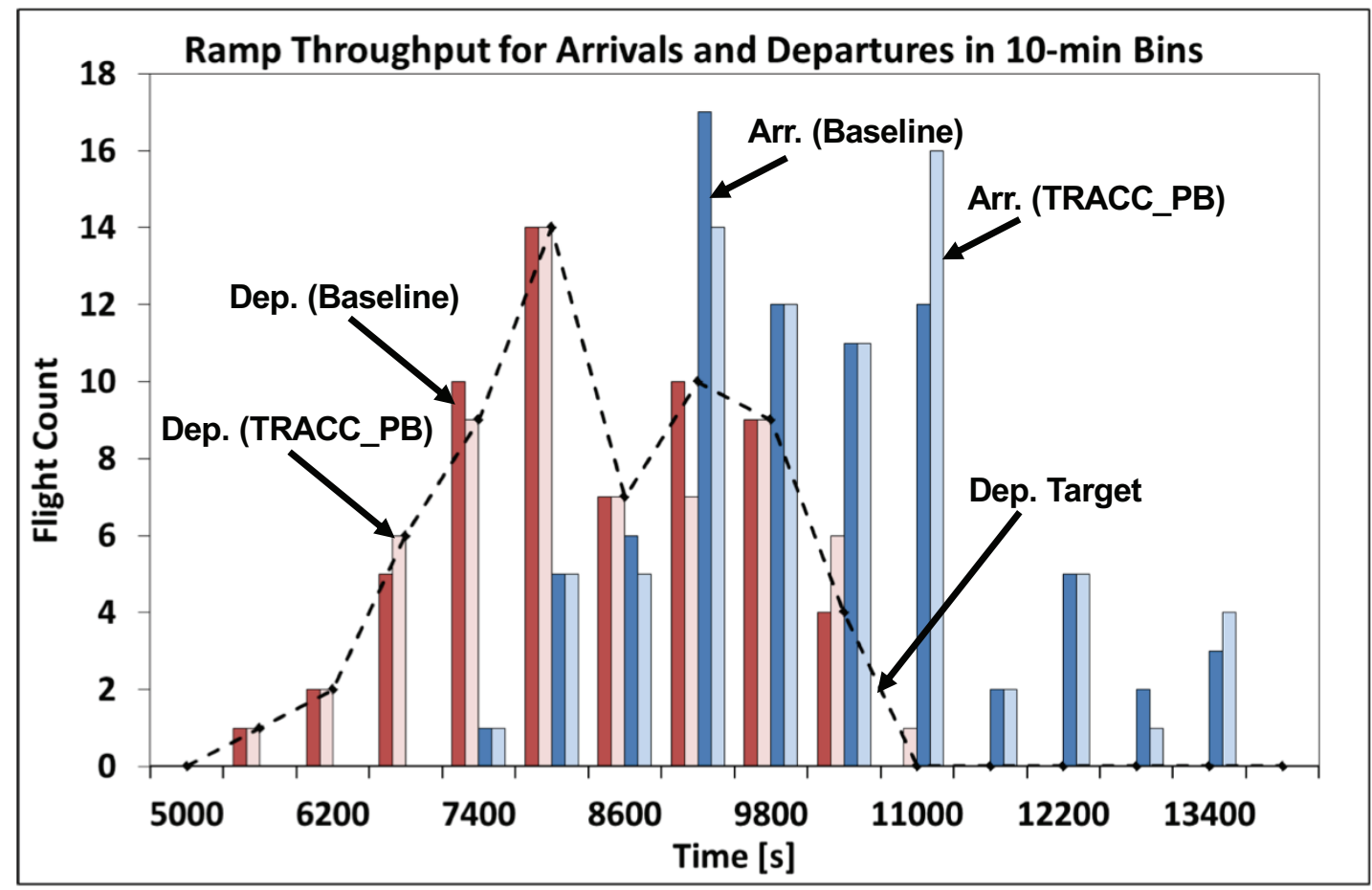

Baseline system is focused on meeting target throughput while TRACC_PB is focused on achieving conflict-free taxi 
DLR.de/fl • Chart 14 > DASC $2018>$ N. Okuniek, Y. Jung • Performance Evaluation of Conflict-Free Trajectory Taxiing in Airport Ramp Area Using Fast-Time Simulations > September 23-27, 2018, London, UK

\section{Results and Analysis - Ramp Taxi \& Gate Hold Times}

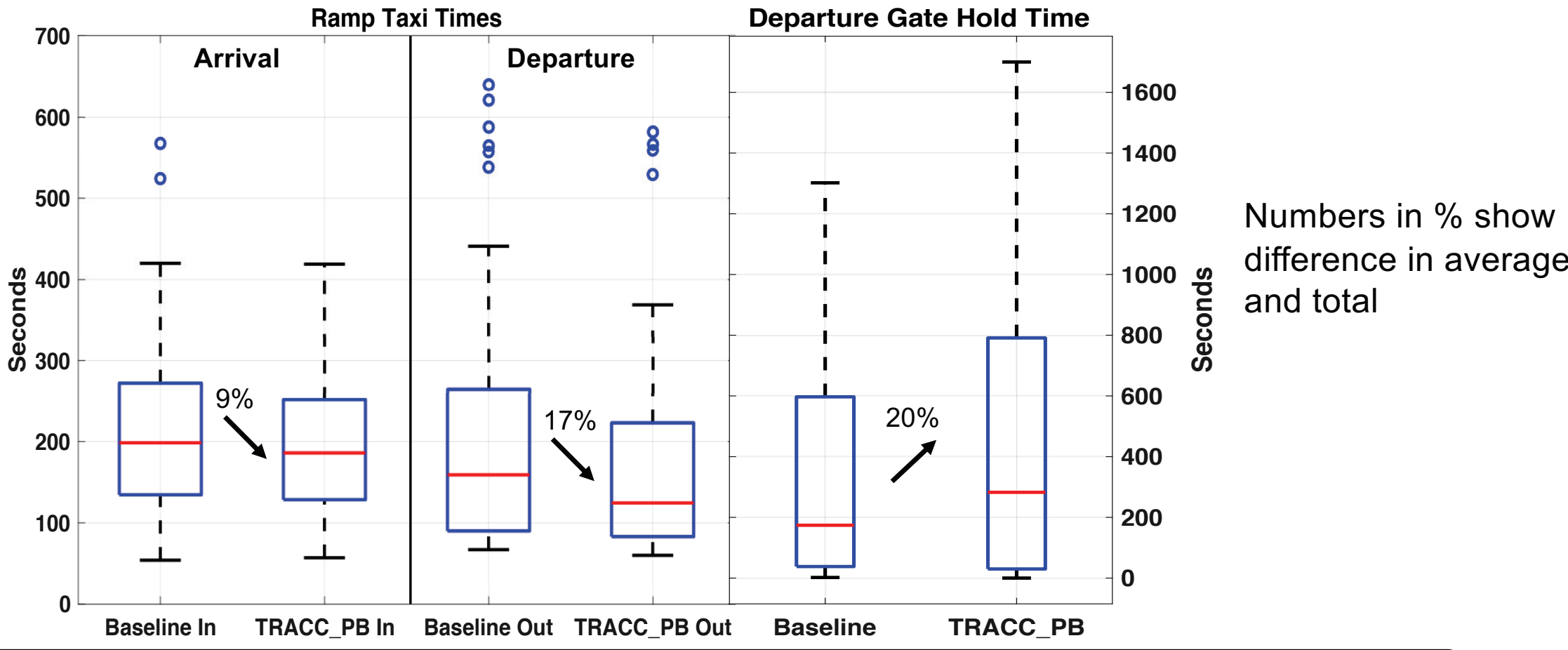

- TRACC_PB reduces ramp taxi time through optimized speed profile enabling conflict-free taxi

- TRACC_PB holds aircraft longer at the gate than the Baseline 


\section{Results and Analysis - TMAT Compliance}

(Actual mov. area entry time - Target mov. area entry time)

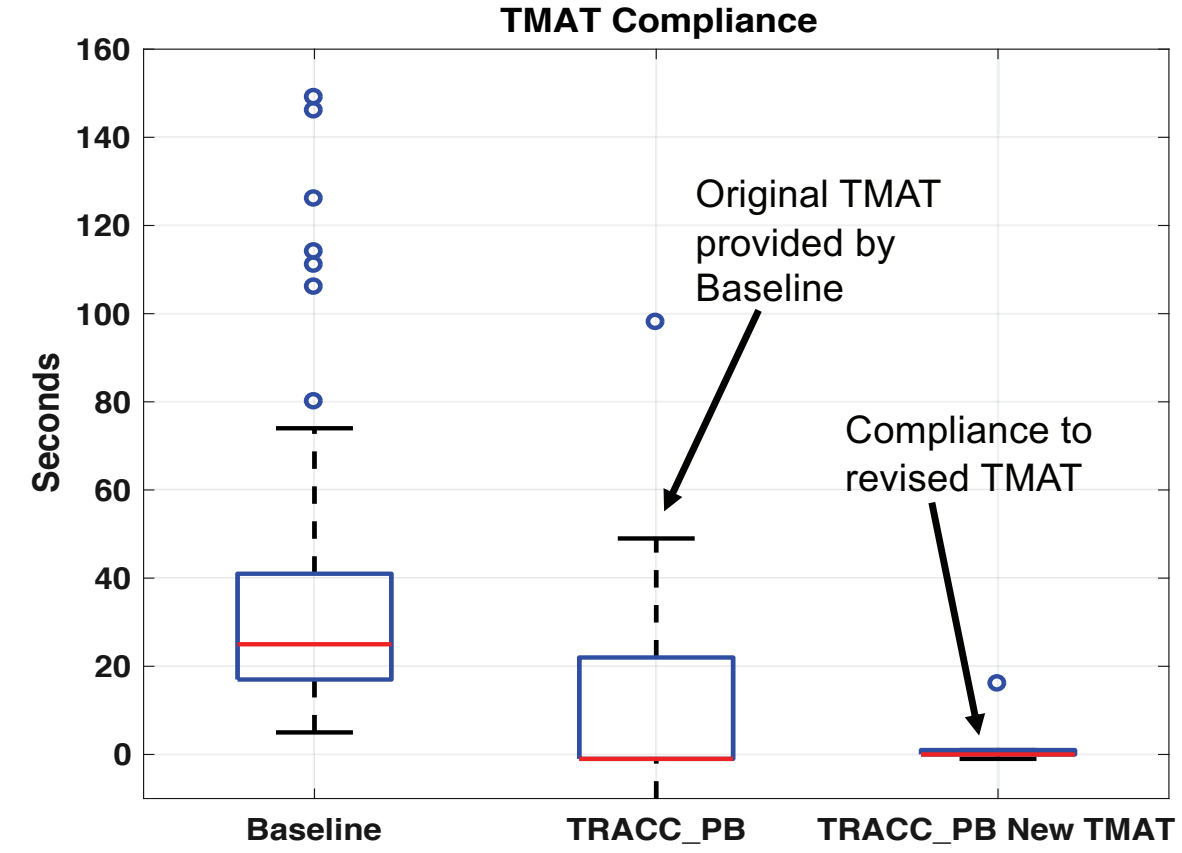

TMAT compliance at various percentiles

\begin{tabular}{|c|r|r|r|r|r|}
\hline Percentile & 25th & 50th & 75th & 80th & 90th \\
\hline $\begin{array}{c}\text { Baseline } \\
\text { (Sec) }\end{array}$ & 17 & 25 & 41 & 43 & 87 \\
\hline $\begin{array}{c}\text { TRACC_PB } \\
\text { (Sec) }\end{array}$ & 0 & 0 & 22 & 44 & 279 \\
\hline
\end{tabular}

TRACC_PB's TMAT compliance outperforms the Baseline in $\sim 80 \%$ of situations

AMAT $=$ Actual Movement Area entry Time 


\section{Results and Analysis - TMAT Compliance (Actual mov. area entry time - Target mov. area entry time)}

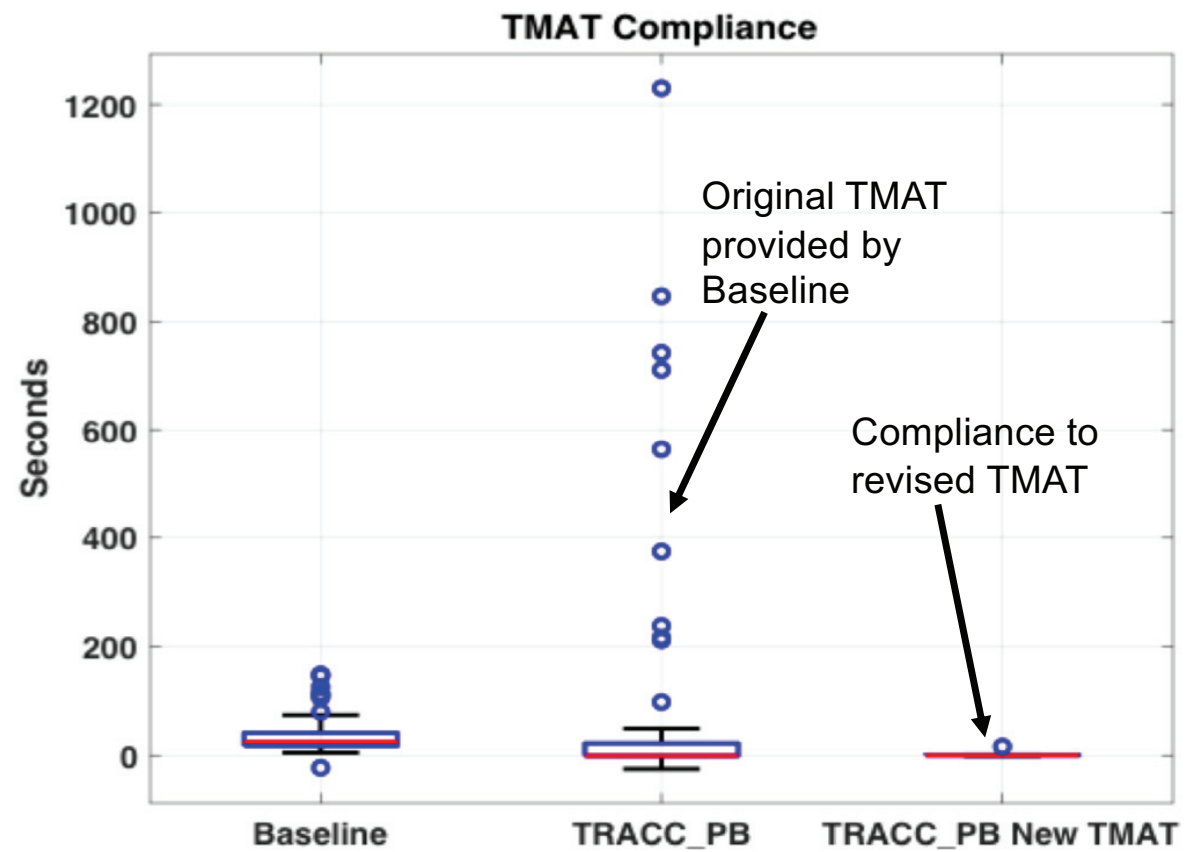

TMAT compliance at various percentiles

\begin{tabular}{|c|r|r|r|r|r|}
\hline Percentile & 25th & 50th & 75th & 80th & 90th \\
\hline $\begin{array}{c}\text { Baseline } \\
\text { (Sec) }\end{array}$ & 17 & 25 & 41 & 43 & 87 \\
\hline $\begin{array}{c}\text { TRACC_PB } \\
\text { (Sec) }\end{array}$ & 0 & 0 & 22 & 44 & 279 \\
\hline
\end{tabular}

Outliers of TRACC_PB's TMAT compliance is related to longer gate hold times $(<1700$ secs $)$

AMAT $=$ Actual Movement Area entry Time 


\section{Results and Analysis - Environmental Benefits (Departures)}

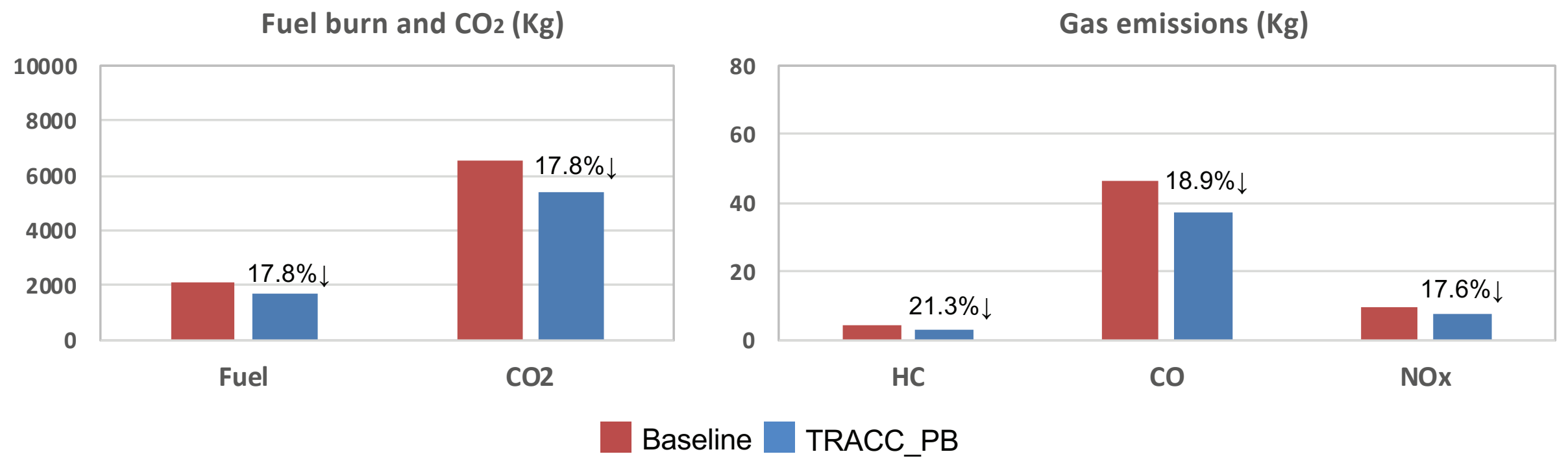

- Metrics were computed following ICAO standards with the assumption of both engines running

- TRACC_PB results showed better performance in fuel and emissions than the Baseline 


\section{Results and Analysis - Environmental Benefits (Arrivals)}

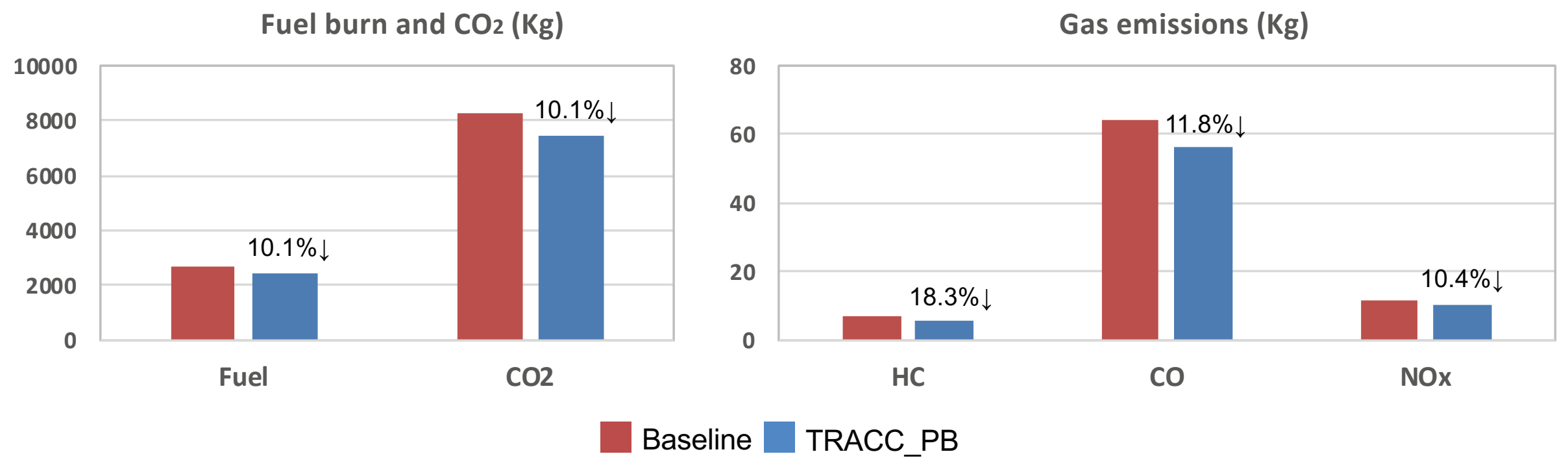

- Metrics were computed following ICAO standards with the assumption of both engines running

- TRACC_PB results showed better performance in fuel and emissions than the Baseline 


\section{Summary}

- Both Baseline and TRACC_PB systems

- used gate holding to shift excess taxi time in the ramp to the gate

- sought to comply with target movement area entry times

- The conflict-free taxi solution by TRACC_PB led to less taxi times, longer gate holding, and less environmental impact than the Baseline system

- TRACC_PB result showed a slight decrease in ramp departure throughput due to larger gate holding

- Both systems showed a good compliance to target movement area entry time

- Meets current Surface Collaborative Decision Making (CDM) suggestion: \pm 5 min around target

- However, TRACC_PB result shows a better compliance than Baseline in $80 \%$ of situations
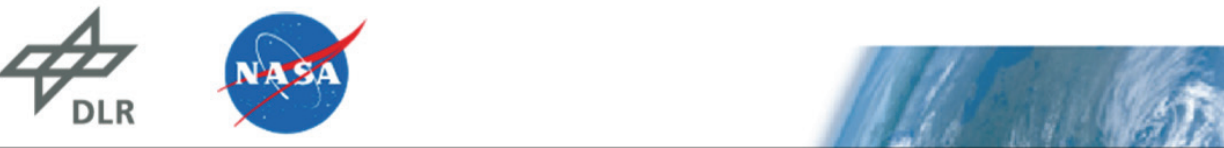


\section{Suggested Future Research}

- Investigation of uncertainties in taxi process to provide robust taxi schedules

- Investigation of off-nominal conditions

- Flight deck analysis will be refined to balance the research between ground-based decision support tools and the flight deck automation 


\section{Thank you!}

\section{Contact}

Nikolai Okuniek

Institute of Flight Guidance

German Aerospace Center (DLR)

nikolai.okuniek@dlr.de
Contact

Dr. Yoon C. Jung

Aviation Systems Division

NASA Ames Research Center

yoon.c.jung@nasa.gov

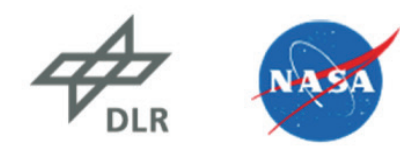




\section{Back up slides}

$\mathscr{H}_{\mathrm{DLR}}$ Naga 


\section{Results and Analysis - Ramp Throughput}

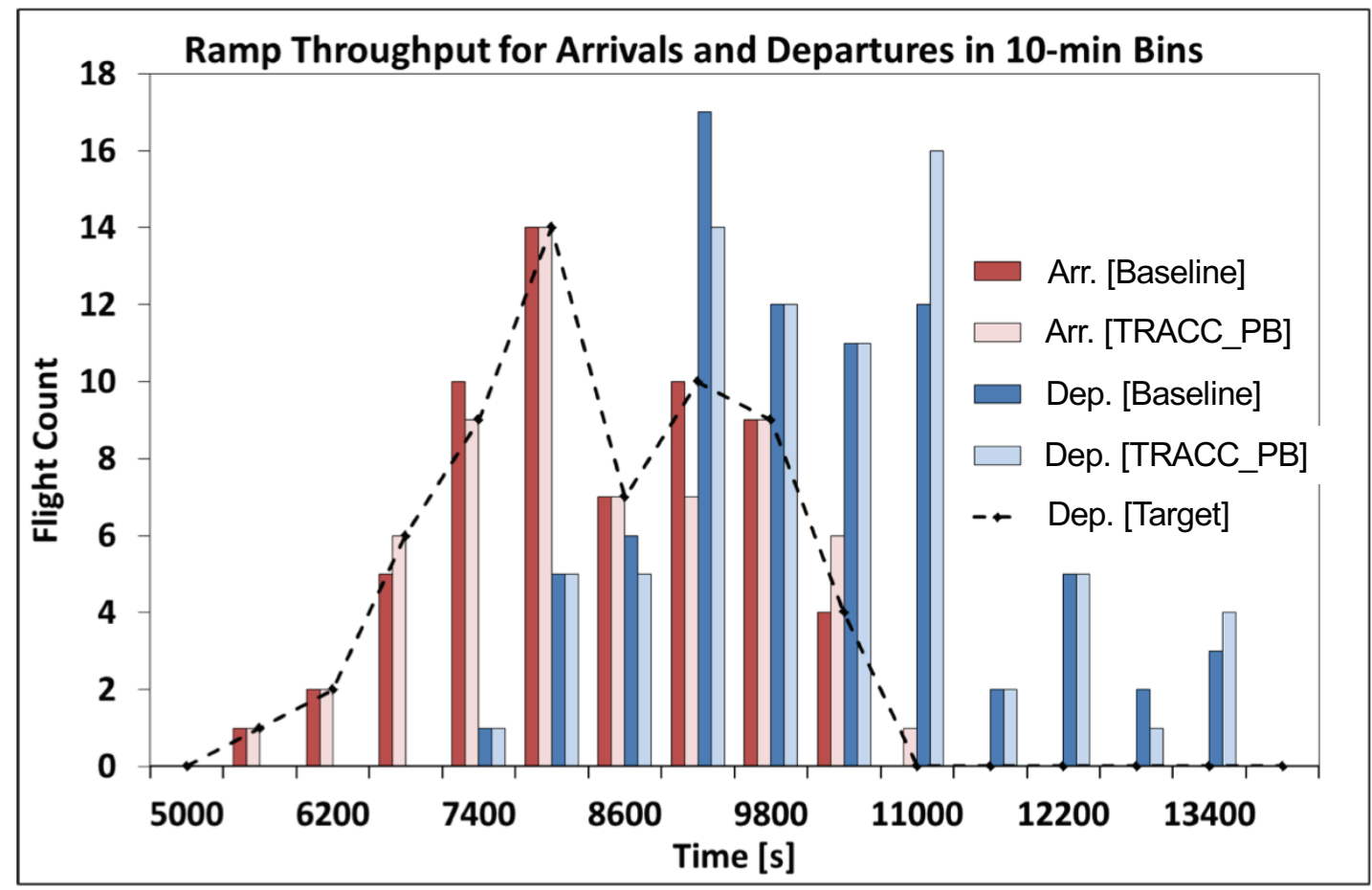

Baseline system is focused on meeting target throughput while TRACC_PB is focused on achieving conflict-free taxi 


\section{Results and Analysis - Ramp Departure Flight Count}

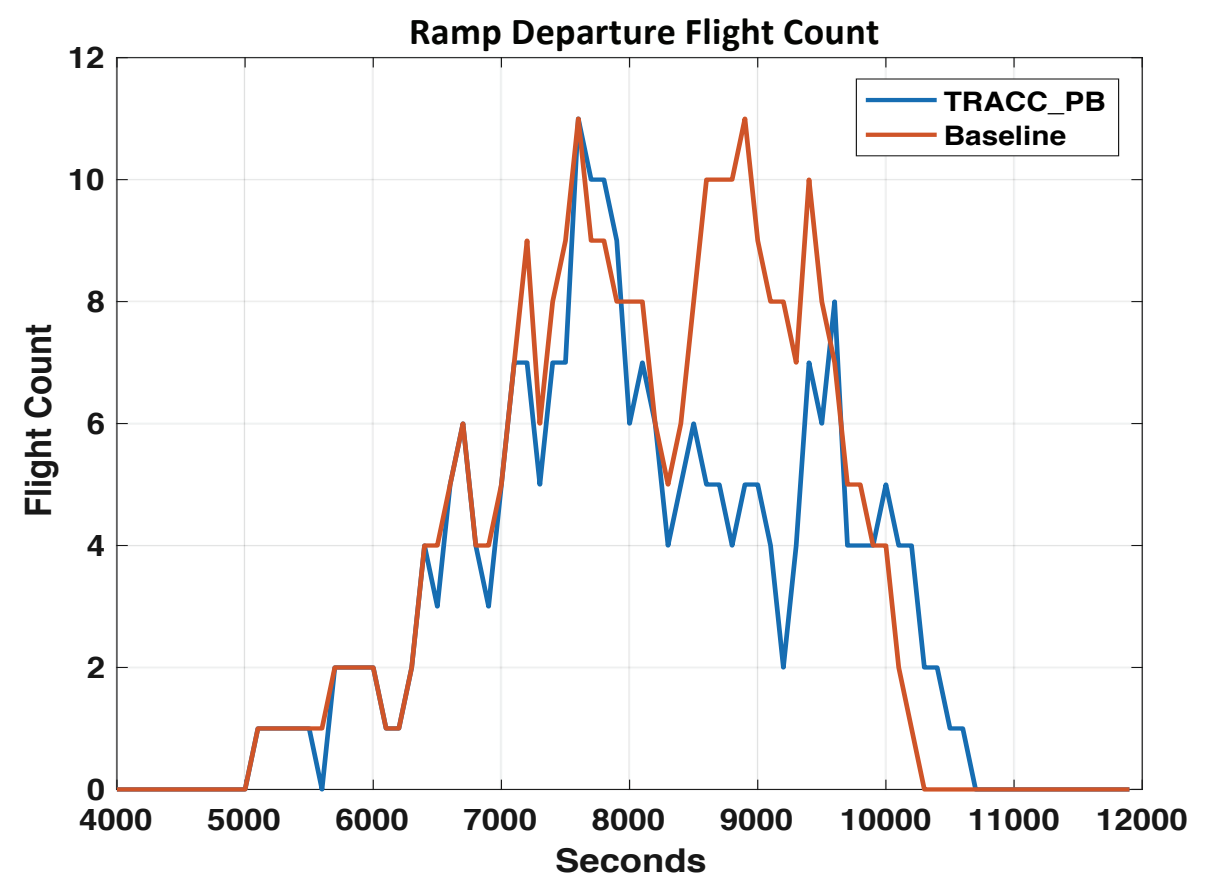

TRACC_PB's conflict-free trajectories contribute to less ramp congestion, but takes more time to flush out departures 


\section{Results and Analysis - Environmental Benefits}

\begin{tabular}{|l|l|l|l|l|l|l|}
\hline & \multicolumn{5}{|c|}{ Departures } & \multicolumn{3}{c|}{ Arrivals } \\
\hline Fuel $(\mathrm{Kg})$ & Baseline & TRACC_PB & Difference \% & Baseline & TRACC_PB & Difference \% \\
\hline $\mathrm{CO}_{2}(\mathrm{Kg})$ & 65116.05 & 1739.68 & $17.8 \downarrow$ & 2680.64 & 2410.19 & $10.1 \downarrow$ \\
\hline $\mathrm{HC}(\mathrm{Kg})$ & 4.27 & 3.36 & $21.3 \downarrow$ & 6.84 & 5.59 & $18.3 \downarrow$ \\
\hline $\mathrm{CO}(\mathrm{Kg})$ & 46.28 & 37.55 & $18.9 \downarrow$ & 64.13 & 56.56 & $11.8 \downarrow$ \\
\hline $\mathrm{NO}_{\mathrm{x}}(\mathrm{Kg})$ & 9.42 & 7.76 & $17.6 \downarrow$ & 11.88 & 10.65 & $10.4 \downarrow$ \\
\hline
\end{tabular}

- Metrics were computed following ICAO standards with the assumption of both engines running

- TRACC_PB results showed better performance in fuel and emissions than the Baseline 